\section{Minimal intervention dentistry: part 2. Caries risk assessment in adults}

\author{
M. Fontana ${ }^{1}$ and C. Gonzalez-Cabezas ${ }^{2}$
}
IN BRIEF
- Reviews the importance of caries risk assessment in adults.
- Demonstrates how risk assessment can be incorporated in everyday clinical practice.
- Presents a clinical case report of a patient with a moderate to high risk of dental caries.

Risk-based, patient-centred decision-making, supported by best available evidence is an essential component for the correct prevention, control and management of dental caries. This article reviews the importance of caries risk assessment in adults as a prerequisite for appropriate caries preventive and treatment intervention decisions. A clinical case will be used to demonstrate how risk assessment can be easily incorporated in everyday clinical practice, using information readily available in the dental-medical history and clinical examination.

\section{INTRODUCTION}

Risk-based prevention and disease management have been recognised as the cornerstones of modern caries management ${ }^{1-3}$ and are essential components of the minimal (minimum) intervention (MI) approach. MI stresses a preventive philosophy with individualised risk assessment, accurate and early detection of lesions

\section{MINIMAL INTERVENTION DENTISTRY}

1. From 'compulsive' restorative dentistry to rational therapeutic strategies

2. Caries risk assessment in adults

3. Paediatric dental care: prevention and management protocols using caries risk assessment for infants and young children

4. Detection and diagnosis of initial caries lesions

5. Atraumatic restorative treatment (ART) - a minimum intervention and minimally invasive approach for the management of dental caries

6. Caries inhibition by resin infiltration

7. Minimally invasive operative caries management: rationale and techniques

This paper is adapted from: Featherstone J D B, Doméjean S. Le concept d'Intervention minimale en cariologie. De la dentisterie restauratrice 'compulsive' aux stratégies thérapeutiques raisonnées. Réalités Cliniques 2011; 22: 207-12.

${ }^{*}-2$ Associate Professor, Department of Cariology, Restorative Sciences and Endodontics at the University of Michigan School of Dentistry, Michigan 48109, USA ${ }^{*}$ Correspondence to: Margherita Fontana

Email: mfontan@umich.edu; Tel: +1 7346471225

Accepted 21 June 2012

DOI: $10.1038 /$ sj.bdj.2012.1008

${ }^{\circledR}$ British Dental Journal 2012; 213: 447-451 and efforts to remineralise non-cavitated lesions with the prompt provision of preventive care in order to minimise operative intervention. When operative intervention is required unequivocally, typically for an active cavitated lesion, the procedure used should be as minimally invasive as possible. ${ }^{4,5}$ This risk-based clinical decision-making for caries management in everyday clinical practice should be based on the best available evidence whilst taking into account the dentist's knowledge and expertise and focusing on the needs and desires of the patient. ${ }^{6}$

Opponents of this strategy maintain that it is difficult to identify such patients accurately, and that even if we could, the evidence for preventive measures on highrisk individuals is still not very strong. All of this is in part true; however, we contend that when the wellbeing of the patient is considered, it is more important to carry out a risk assessment incorporating the best available evidence than just doing nothing due to lack of strong evidence. Others allege that similar preventive measures should be administered to the whole population, regardless of the risk. However, for the current environment of increasing healthcare costs and resource constraints, targeted healthcare delivery has become paramount, depending profoundly on risk assessment. If a clinician practices in an environment in which all patients have a similar risk of caries, then we agree that doing individual risk assessments would add no value to the clinician or the patient. However, dental caries is unequally distributed in most populations around the world, with a small percentage of individuals carrying the heavier burden of caries disease. ${ }^{7}$ For most dentists in private practice, it becomes imperative to be able to identify a patient's risk status in order to be able to develop the most cost-effective treatment strategy for that individual. Due to the multifactorial nature of the caries process, and the fact that the disease is dynamic (for example, lesions can progress and/or regress), studies on risk assessment tend to be complex, with a multitude of variables challenging the prediction at different times during the life of an individual. ${ }^{8}$ Most studies on risk assessment have been conducted in children (see later article in this $B D J$ series) and there is very little evidence from adults or the elderly to help guide practitioners on how to apply risk assessment models to adult populations. ${ }^{2,9}$

Therefore, caries risk assessment should be useful in the clinical management of dental caries by helping to:

- Determine lesion activity

- Estimate the degree of risk so the intensity of the treatment (for example, fluoride concentration and delivery method) and frequency of appointments can be customised

- Identify the main aetiological agents contributing to the current disease that might be targeted in the management 
of the disease (for example, diet control)

- Establish the need for additional diagnostic procedures (for example, salivary flow rate/buffering measurements)

- Formulate the best restorative treatment (care) plan for this patient (for example, dental material selection)

- Enhance the overall prognosis of the patient

- Appraise the efficacy of the caries management plan established at recall visits.

This paper reviews the importance of caries risk assessment in adults as a prerequisite for appropriate caries preventive and treatment intervention decisions.

\section{RISK INDICATORS}

Traditionally, caries disease indicators have been defined as clinical observations that tell about the past caries history and activity. They are indicators or clinical signs that there is disease present or that there has been recent disease. ${ }^{1}$ These indicators also include variables that say nothing about what caused the disease or how to treat it, but that are related to disease experience (for example, socioeconomic status, education). Caries experience is an illustration of an indicator that shows how the host copes with the biological activity. ${ }^{10}$ However, as before, others have defined risk indicators as factors established only in cross-sectional studies as being associated with the disease without any longitudinal validation. ${ }^{11}$

\section{Caries experience}

The strongest risk indicator is past caries experience and current lesion activity. ${ }^{8,12,13}$ As a predictor it is simple, inexpensive and fast, as it requires a dental examination only. If approximal lesions are included in the risk analysis, then radiographs, especially radiographic follow-up of existing lesions, would enhance the diagnosis. Past caries experience summarises the cumulative effect of all risk factors and protective factors to which an individual has been exposed over a lifetime. However, exposure to risk factors may change over a lifetime, and this affects the predictive power of this indicator making it less than 100\% accurate. Risk factors that lead to the patient's past caries experience might have changed over their lifetime and sometimes we are simply seeing the consequences of the disease that occurred years ago. Nevertheless, as mentioned earlier, epidemiological studies have shown a positive strong correlation between past caries experience and future caries development, which is why all available risk tools include this indicator very prominently. In adults, there is also a clear association between coronal caries and the risk of developing root caries ${ }^{14,15}$

It is important to assess not only cavitated lesions, but non-cavitated lesions also. If lesions are present it is imperative to decide whether they are likely active or arrested, as this will influence the analysis of future risk. Presence of current activity would indicate a high likelihood that if conditions do not change, activity will continue in the future.

Another important aspect to take into consideration is the site specificity of the disease. Caries lesions develop in areas where dental plaque is stagnant for long periods of time. For younger individuals, the occlusal surfaces of posterior teeth are by far the areas more frequently affected by the disease, but for older patients, however, this might not be the case. Frequently, older adults have very few or no fissures without restorations in posterior teeth reducing the risk of caries in those high risk surfaces when restorations in good condition are present. However, restorations with significant defects will accumulate significant amounts of plaque biofilm, increasing the risk at that specific site. In these people, newly exposed root surfaces and defective restorations need to be carefully examined and have their risk for caries determined.

\section{Socio-demographic indicators}

Although socioeconomic status is a stronger predictor of caries risk in children than in adults, it is still important in adults ${ }^{13,16}$ However, because dental caries generally is more prevalent in lower than higher socioeconomic classes, the dentist should consider the social environment of the patient (for example, education, income, occupation etc) as available to him/her through the medical history, in the analysis of caries risk.

\section{Notes}

- The best indicators of caries risk can be attained easily from data obtained at periodic dental examinations and do not require additional testing routinely. This is, of course, very encouraging for every day clinical practice

- The fact that previous caries experience is such a strong predictor is, from a disease management perspective, a less than desirable outcome, considering the fact that the disease is actually manifest before it can be accurately predicted, and the ultimate goal of caries management is to prevent disease in the first case

- The fact that the existence of recent restorations is one of the greatest indicators of risk for the development of new caries lesions only proves that the act of treating the caries lesion surgically does little to reduce the risk of developing the next lesion ${ }^{9,13,17}$

- In a systematic review, Zero et al. concluded that for caries prediction in permanent teeth in adults, past caries experience was the best predictor, followed by education and marital status, probably because these factors influenced attitudes towards oral health $^{9}$

- It is very important to realise that the assessment of all risk factors not only allows a more complete assessment of future risk of disease, but most importantly identifies the aetiological factors responsible for the disease in a particular patient. $^{2}$

\section{RISK FACTORS}

Traditionally, a risk factor plays an essential role in the aetiology of the disease, while a risk indicator is indirectly associated with the disease. In other words, caries risk factors are the biologic reasons, or factors, that have caused or contributed to the disease, or will contribute to its future manifestation on the tooth (for example, bacteria, diet etc). ${ }^{8}$

\section{Genetics}

Although this is the only factor that cannot be measured currently in clinical practice, it is important to highlight that even when there is still much to learn about the genetic-environmental relationships in dental caries aetiology and risk assessment, the amount of evidence relating to genes and caries experience has increased 
significantly in the last decade. As reviewed recently by Wright, genes have been identified linking tooth development, salivary function and diet/taste to caries risk or protection. ${ }^{18}$ This is very important because as the understanding of genetics associated with caries risk increase, so does the future possibility of using salivary diagnostics based on genetic scans to develop either better risk assessment tools or to better target specific interventions that would improve the oral and general health of at-risk populations.

\section{Saliva}

It is well established that saliva plays an important role in the health of soft and hard tissues in the oral cavity. Dentists can assess several salivary parameters as related to caries risk, but the most common ones include salivary flow rate, buffering capacity and $\mathrm{pH}$. Chronically low salivary flow rate (that is, true hypo-salivation) has been found to be one of the strongest salivary indicators for an increased risk of dental caries. ${ }^{19}$ Apart from this scenario, the caries prediction of saliva parameters is modest to low, and thus hard to justify routinely in dental practice for every patient.

Oral complications as a result of salivary gland hypofunction include altered oral sensations, taste dysfunction, mucosal dryness resulting in infection and tooth wear due to abrasion, among other factors. Pain and diminished quality of life are also common complaints associated with salivary hypofunction. ${ }^{20}$ Many dentists tend to rely on the complaint of dry mouth or xerostomia to diagnose hyposalivation. Unfortunately, subjective complaint of xerostomia often does not correlate with objective findings of reduced salivary flow rate. Fox et al. ${ }^{21}$ recommended that dental care professionals ask their patients the following questions: does your mouth feel dry when eating a meal? Do you sip liquids to aid swallowing dry foods? Do you have difficulty swallowing any foods? Does the amount of saliva in your mouth seem to be too little, too much or do you not notice it? A positive answer to any of these questions should prompt consideration as to how long the patient has experienced the problem, whether or not an increased caries experience has resulted and lead to an objective measurement of salivary flow rate. Other questions, such as 'is your mouth dry?' are not predictive at all, since most people have dry mouths in the mornings due to the normal decrease in salivary flow rate which occurs during sleep.

Many current medications (for example, psycho-pharmaceutical products) reduce the flow rate of saliva in a percentage of the population using them, and therefore may affect their caries risk. Also, certain diseases, especially those related to decreases in salivary flow rate, such as Sjögren's syndrome and uncontrolled diabetes, can increase the risk of caries.

\section{Bacteria}

Dental caries is a microbial disease in which the aetiological agents are normal constituents of the dental plaque biofilm that cause problems only when their pathogenicity and proportions change in response to environmental conditions.

It is clear that without any plaque biofilm there would be no caries. However, most patients do not remove plaque effectively from areas at high risk. The principle of microbial testing in clinical practice is the thought that people with high numbers of cariogenic bacteria are at higher risk for developing future lesions and, as such, should be treated, however:

- Most plaque indices are ineffective predictors of future caries because dental caries typically develops in fissures and interproximal areas, while most plaque indices were developed to evaluate periodontal disease or gingivitis based on smooth surface scores

- To solely evaluate the effectiveness of mechanical cleaning is difficult because tooth brushing usually involves a dentifrice with fluoride. However, it is known that any conditions that compromise the long-term maintenance of good oral hygiene, and for which the patient has not been able to show the ability to maintain plaque-free, are positively associated with caries risk

- Salivary bacterial tests have existed for several years and are based on the premise that saliva levels represent levels in the oral biofilm. As one of the primary aetiologic agents of dental caries, mutans streptococci and lactobacilli historically have captured the greatest interest among researchers and clinicians. There is great controversy in the literature regarding the accuracy of salivary tests for mutans streptococci and lactobacilli in predicting future caries in adults. ${ }^{13}$ Several tests exist in the market to measure salivary bacteria based on culturing. These tests have disadvantages because they require incubators, many enumerate bacteria in saliva only (not in plaque), and in general they correlate poorly in adults with future caries risk. However, they may be useful to motivate and monitor oral hygiene, assess the effectiveness of oral antimicrobials and monitor dietary changes.

Newer tests are available that measure site-specific plaque $\mathrm{pH}$, or measure bacterial load using either monoclonal antibodies, or responses to ATP bioluminescence and although useful for patient education, many of these have not been validated longitudinally yet for risk assessment.

\section{Diet}

Sugar exposure is an important aetiological factor in caries development. Due to the wide use of fluoride and its effect in lowering the incidence and rate of caries, it is difficult to show a strong, clear-cut, positive association between a person's total sugar consumption and his/her caries development. Thus, for example, selfreported sugar intake seems to have little value at identifying, by itself, patients at risk. However, diet is one of the main drivers of caries activity, and recognising the behaviours that are placing the patient at risk may be very important for caries prevention and management. ${ }^{22}$

Other dietary considerations include the retentiveness of the food, frequency of consumption (this being the most important), consumption between meals, the presence of protective factors in foods (for example, calcium, fluoride) and the type of carbohydrate. Although sugar in liquid form (for example, soft drinks) is less cariogenic than sugar in solid form (for example, sweets), excessive frequent consumption of soft drinks remains a major risk factor that may be partly responsible for the high rate of caries in teenagers and young adults in many parts of the world. As a reminder, 
starches are considered less cariogenic than the simple sugars sucrose, glucose and fructose, with sucrose possibly being the most cariogenic due to its unique role in the production of extracellular polysaccharides.

\section{Others}

Mental and/or physical disabilities that affect regular oral hygiene or which require a more frequent carbohydrateenriched diet also may affect the individual's risk. Additionally, enamel defects, such as hypoplasia, have also been related to increase caries risk, especially in children.

\section{PROTECTIVE FACTORS}

Caries protective factors are biologic or therapeutic factors/measures that can collectively offset the challenge presented by caries risk factors. The more severe the risk factors, the higher the protective factors need to be to keep the patient in balance or to reverse the caries process.

We recommend that risk assessment in adults include an analysis of risk indicators, risk factors and protective factors. To determine the risk status of a patient we must balance the presence of risk factors that the patient currently has, plus risk indicators of past and current disease history and weigh that against the protective factors that the patient is exposed to. ${ }^{1}$ Although the clinician should analyse all protective factors the patient is exposed to, of great importance is the exposure to fluorides. The widespread use of fluoride has reduced dramatically the prevalence of dental caries and the rate of the progression of carious lesions. ${ }^{23}$ Its use, which should be considered one of the most important protective factors when assessing a patient's caries risk, allows more conservative management strategies for the prevention and treatment of dental caries. The frequency of fluoride exposure is one of the most important considerations when considering its use as a management and preventive strategy to control dental caries. This is because the main mechanism by which fluoride prevents against caries is by enhancing remineralisation. When does remineralisation need to happen? Every time there is demineralisation. The dental care professional should consider all fluoride sources to which the patient is exposed, for example, fluoridated drinking water, foods/ drinks, home topical fluoride products and periodic professional fluoride exposures. The dentist should then determine if this history/pattern of fluoride exposure has arrested the appearance or progression of carious lesions (incipient or cavitated) over time. If new lesions have appeared or existing lesions have progressed, then the patient's fluoride exposure is inadequate. As the risk is increased, so should be the level of fluoride exposure, both at home and in office. ${ }^{2,24}$ In addition, fluoride use should be individually determined for each patient based on age, physical abilities, health awareness and attitude.

\section{CASE REPORT}

For the patient presented in the following case report (Figs 1-3) it is not necessary to complete a complicated and long risk assessment form or to measure risk factors using chair-side bacterial or salivary tests available in the market to determine that the patient is at elevated risk for dental caries. The presence of clear clinical signs of disease activity (presence of active caries lesions) can be used to help predict a higher risk of future disease progression. But, what if there had not been any signs of active disease? Does this mean the risk of developing caries lesions in the next few years is low?

\section{Risk indicators}

\section{Caries experience}

Caries experience has been high and there are numerous lesions present that appear active; therefore, we should classify this patient, as explained later on, as moderate to high risk. Although this information is helpful, we still do not know the specific reasons behind the caries experience of this patient.

\section{Socio-demographic indicators}

The patient is female, 63 years old, of lower middle class, divorced, living independently for the last seven years, but under a lot of financial stress. Although none of these factors are a strong predictor of her future risk, they point to an environment which may be conducive, for example, to difficulty accessing care as frequently as needed.

\section{Risk factors}

\section{Saliva}

In the present case, there were no signs or

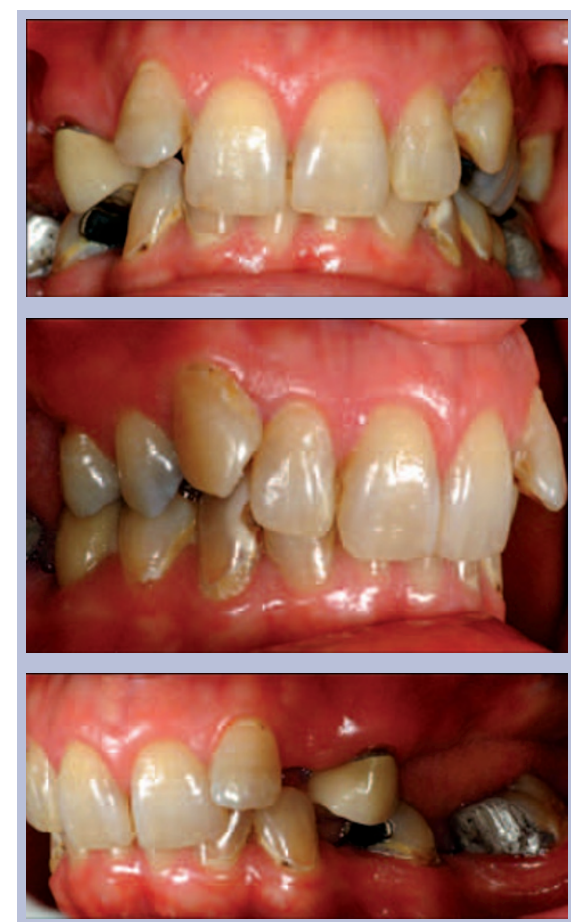

Figs 1-3 Clinical photographs presenting patient with a moderate to high risk of dental caries

symptoms of salivary dysfunction. None of the conditions, which could affect the salivary flow rate, such as Sjögren's syndrome, uncontrolled diabetes or medications, were present in the patient described.

\section{Bacteria}

This patient presented mature, stagnant plaque around most cervical and interproximal areas, with gingivitis associated with it. This clinical observation suggests these are risk areas for caries development in this patient.

\section{Diet}

When initially questioned, the patient did not think she had a high sugar-rich diet. However, her active lesions suggested there must be a current dietary factor in play. Upon closer examination, she admitted to drink very frequently throughout the day coffee with sugar, usually with a cookie or two. Although not an unusual behaviour for some people, the combination of this habit with presence of stagnant mature plaque and lack of protective factors (discussed next) increases the risk of the patient.

\section{Protective factors}

The patient used to brush twice a day with a dentifrice with fluoride; however, since 
her divorce seven years ago she is brushing less than once a day. In addition, she has received no in-office fluoride treatments over the last two decades. Even though she may have had an appropriate level of fluoride exposure at one time, currently this level is not enough to balance out the plaque and dietary factors she is being challenged with.

\section{Re-evaluation}

In order to provide frequent counselling and exposure to in-office fluoride, the recall interval was set at four months.

\section{CONCLUSIONS}

Considering the current understanding of the caries disease process, we propose the following factors, whether appearing singly or in combination, would yield a moderate to high risk assessment of caries (as in the case presented in this paper): the development of new caries lesions, the presence of active lesions and the placement of restorations due to active disease since the patient's last examination (assuming a one to two-year lapse between the previous and current appointment). Finally, of greatest importance is that for moderate and high risk individuals; once you have determined they are at risk and have identified the reasons why, the dental team then has to decide what is the simplest and most likely successful strategy, both from the biological and behavioural perspective, for managing the caries disease in that particular patient. This includes a decision of both preventive and restorative approaches.

We also propose that a low caries risk assessment be based on the following factors: no caries lesion development or progression for a period of one to three years; amount of plaque accumulation; frequency of the patient's sugar intake; presence of salivary problems; behavioural or physical disability changes; history of fluoride exposure and pattern of fluoride usage.

A dentist's overall subjective impression of the patient has a relatively good predictive value for caries risk, ${ }^{25}$ but it is unclear how this information is incorporated into everyday clinical practice. Recent concepts in caries management have not been largely accepted: a recent survey of clinical practices within a U.S. practice-based research network suggests that a significant proportion had yet to adopt treatments based on assessment of caries-risk. ${ }^{26}$ Another practice-based study showed that in France decision-making in caries management does not only depend on pathophysiology. ${ }^{27}$ Thus, a more objective, easy to implement, and validated risk assessment instrument is desirable and this is reflected in the multiple risk assessment tools that have been developed during the last few years. Examples for adults include the American Dental Association's caries risk tool for adults, ${ }^{28}$ the caries management by risk assessment (CAMBRA) tool for adults, ${ }^{29}$ and the cariogram. ${ }^{30-32}$

Risk assessment is an essential component of clinical practice for caries management. Most of the information needed is readily available in a properly done health/ dental history and a clinical examination, with the subjective impression of the clinician having been shown to be very useful. Most of the risk indicators or factors provide (either by themselves or combined) only a modest possibility of accurately predicting adults at future risk. However, even with these limitations risk assessment can enhance patient care. The most important factor in predicting future risk is recent caries experience and current disease activity. However, a careful analysis of all risk and protective factors will allow the dental team and patient to understand the specific reasons for the caries disease and thus will allow them to tailor the treatment plan and recall interval specifically to the patient's needs.

The authors would like to thank Claudie DamourTerrasson, publishing director of the Groupe Information Dentaire, Paris France, for the autorisation of translation and publication of the series in the $B D J$ and Dr Sanjay Karunagaran for kindly providing the clinical pictures used in the present article.

1. Featherstone J D. The caries balance: contributing factors and early detection. J Calif Dent Assoc 2003; 31: 129-133.

2. Fontana $M$, Zero D. Assessing patients' caries risk. J Am Dent Assoc 2006; 137: 1231-1249.

3. Featherstone J D. The science and practice of caries prevention. J Am Dent Assoc 2000; 131: 887-899.

4. Fontana M, Young D A, Wolff M S, Pitts N B Longbottom C. Defining dental caries for 2010 and beyond. Dent Clin North Am 2010; 54: 423-440.

5. Reich E, Lussi A, Newbrun E. Caries risk assessment. Int Dent J 1999; 49: 15-26.

6. American Dental Association. Policy of evidence based dentistry. USA: ADA, 2008. Online article available at http://www.Ada.Org/1754.Aspx (accessed October 2012).

7. Edelstein B L. The dental caries pandemic and disparities problem. BMC Oral Health 2006; 6(Suppl 1): S2.

8. Twetman S, Fontana M. Patient caries risk assessment. Monogr Oral Sci 2009; 21: 91-101.

9. Zero D, Fontana M, Lennon A M. Clinical applications and outcomes of using indicators of risk in caries management. J Dent Educ 2001: 65: $1126-1132$.
10. Bratthall D, Hänsel Petersson G. Cariogram - a multifactorial risk assessment model for a multifactorial disease. Community Dent Oral Epidemiol 2005; 33: 256-264.

11. Burt B A. Definitions of risk. J Dent Educ 2001; 65: 1007-1008.

12. Powell L V. Caries prediction: A review of the literature. Community Dent Oral Epidemiol 1998; 26: 361-371.

13. National Institutes of Health (US). Diagnosis and management of dental caries throughout life. NIH Consensus Statement 2001; 18: 1-23.

14. Ritter A V, Shugars D A, Bader J D. Root caries risk indicators: a systematic review of risk models. Community Dent Oral Epidemiol 2010; 38: 383-397.

15. Sánchez-Garcia S, Reyes-Morales H, Juárez-Cedillo T, Espinel-Bermúdez C, Solórzano-Santos F, GarcíaPeña C. A prediction model for root caries in an elderly population. Community Dent Oral Epidemiol 2011; 39: 44-52.

16. Jamieson L M, Mejía G C, Slade G D, RobertsThomson K F. Predictors of untreated dental decay among 15-34-year-old Australians. Community Dent Oral Epidemiol 2009; 37: 27-34.

17. Fejerskov 0 . Changing paradigms in concepts on dental caries: consequences for oral health care. Caries Res 2004; 38: 182-191.

18. Wright J T. Defining the contribution of genetics in the etiology of dental caries. J Dent Res 2010; 89: 1173-1174.

19. Leone C W, Oppenheim F G. Physical and chemical aspects of saliva as indicators of risk for dental caries in humans. J Dent Educ 2001; 65: 1054-1062.

20. Navazesh M. Salivary gland hypofunction in elderly patients. J Calif Dent Assoc 1994; 22: 62-68.

21. Fox P C, van der Ven P F, Sonies B C, Weiffenbach $J \mathrm{M}$, Baum B J. Xerostomia: evaluation of a symptom with increasing significance. J Am Dent Assoc 1985; 110: 519-525.

22. Zero D T. Sugars - the arch criminal? Caries Res 2004; 38: 277-285.

23. Centers for Disease Control and Prevention. Recommendations for using fluoride to prevent and control dental caries in the United States. CDC 2001. Online article available at http://www.cdc. $\mathrm{gov} / \mathrm{mmwr} / \mathrm{preview} / \mathrm{mmwrhtml} / \mathrm{rr} 5014 \mathrm{a} 1 . \mathrm{htm}$ (accessed October 2012).

24. American Dental Association Council on Scientific Affairs. Professionally applied topical fluoride: evidence-based clinical recommendations. J Am Dent Assoc 2006; 137: 1151-1159.

25. Disney J A, Graves R C, Stamm J W, Bohannan H M Abernathy J R, Zack D D. The University of North Carolina Caries Risk Assessment study: further developments in caries risk prediction. Community Dent Oral Epidemiol 1992; 20: 64-75.

26. Riley J L 3rd, Gordan V V, Rindal D B et al. Preferences for caries prevention agents in adult patients: findings from the dental practice-based research network. Community Dent Oral Epidemiol 2010; 38: 360-370.

27. Doméjean-Orliaguet S, Léger S, Auclair C, Gerbaud $L$, Tubert-Jeannin S. Caries management decision: influence of dentist and patient factors in the provision of dental services. J Dent 2009; 37: 827-834.

28. American Dental Association. Caries risk form: (patients over 6 years). ADA, 2008. Online form available at http://www.ada.org/sections/professionalResources/pdfs/topic_caries_over6.pdf (accessed October 2012).

29. Featherstone J D, Domejean-Orliaguet $S$, Jenson $L$, Wolff M, Young D A. Caries risk assessment in practice for age 6 through adult. $J$ Calif Dent Assoc 2007; 35: 703-707, 710-713.

30. Fure S, Zickert I. Incidence of tooth loss and dental caries in 60-, 70-and 80-year-old Swedish individuals. Community Dent Oral Epidemiol 1997; 25: 137-142.

31. Hänsel Petersson G, Fure S, Bratthall D. Evaluation of a computer-based caries risk assessment program in an elderly group of individuals. Acta Odontol Scand 2003; 61: 164-171.

32. Ruiz Miravet $A$, Montiel Company J M, Almerich Silla J M. Evaluation of caries risk in a young adult population. Med Oral Patol Oral Cir Bucal. 2007; 12: E412-E418. 